\title{
Productivity of "collisions generate heat" for reconciling an energy model with mechanistic reasoning: A case study
}

\author{
Rachel E. Scherr and Amy D. Robertson \\ Department of Physics, Seattle Pacific University, Seattle, Washington 98119, USA
}

(Received 11 November 2014; published 18 March 2015)

\begin{abstract}
We observe teachers in professional development courses about energy constructing mechanistic accounts of energy transformations. We analyze a case in which teachers investigating adiabatic compression develop a model of the transformation of kinetic energy to thermal energy. Among their ideas is the idea that thermal energy is generated as a byproduct of individual particle collisions, which is represented in science education research literature as an obstacle to learning. We demonstrate that in this instructional context, the idea that individual particle collisions generate thermal energy is not an obstacle to learning, but instead is productive: it initiates intellectual progress. Specifically, this idea initiates the reconciliation of the teachers' energy model with mechanistic reasoning about adiabatic compression, and leads to a canonically correct model of the transformation of kinetic energy into thermal energy. We claim that the idea's productivity is influenced by features of our particular instructional context, including the instructional goals of the course, the culture of collaborative sense making, and the use of certain representations of energy.
\end{abstract}

DOI: 10.1103/PhysRevSTPER.11.010111

PACS numbers: 01.40.Fk, 01.40.Ha, 01.40.J-

\section{INTRODUCTION}

Canonically incorrect ideas have historically been seen as obstacles to learning. We analyze a series of classroom events in which a canonically incorrect idea-that individual particle collisions in an ideal gas generate "heat"plays a productive role in classroom discourse, initiating reasoning that eventually leads to a canonically correct model of the transformation of kinetic energy into thermal energy. We connect our case to existing literature on the role of canonically incorrect ideas in learning, adding to this literature by offering evidence that the productivity of a canonically incorrect idea is not only a property of the idea itself, but also depends on features of the particular instructional context: in our case, specific instructional goals, a culture of collaborative sense making, and certain representations for energy. In doing so, we aim to encourage physics instructors and physics education researchers to frame student ideas as potentially productive, seeking the seeds of correctness in their students' thinking.

We first review previous research about the role that canonically incorrect ideas play in learning, including the commonly reported idea that collisions between ideal gas particles generate thermal energy (Sec. II). We then situate this manuscript in our broader research and instructional context, sharing details of the course in which the events we describe take place and describing our data collection,

Published by the American Physical Society under the terms of the Creative Commons Attribution 3.0 License. Further distribution of this work must maintain attribution to the author(s) and the published article's title, journal citation, and DOI. episode selection, and analytical framework (Sec. III). We go on to describe what we mean by "conceptual model for energy" and "mechanistic reasoning," the two commitments that the learners in our episodes seek to reconcile (Sec. IV). Next, we illustrate and analyze a progression of learner discourse about adiabatic compression that begins with the idea that collisions generate heat (Sec. V). We make the case that the productivity of the idea that collisions generate heat is influenced by features of our instructional context (Sec. VI). We close with a discussion that responds to possible counterarguments and presents some instructional implications of our analysis.

In what follows, learners (in this case, secondary teachers) frequently use the term "heat" ambiguously, sometimes seeming to refer to thermal energy and sometimes to warmth. An association of heat with the temperature of an object is common in everyday speech, in nonphysics textbooks, and in standards documents $[1,2]$. However, such an association is not aligned with disciplinary norms in physics, in which the energy associated with temperature is often termed "thermal energy" and in which the term heat refers to energy transfer from a body at higher temperature to one at lower temperature. Learners in our courses often use heat or "heat energy" to refer to a form of energy indicated by temperature (what we call thermal energy), rather than a transfer of energy driven by temperature difference (what we call heat) [2-5]. In characterizing learners' ideas, we sometimes adopt their language, even though it is canonically incorrect. Our rationale for doing so is that to rephrase their idea as "collisions generate thermal energy" may suggest a model of energy that is not necessarily shared by the learners: thermal energy, being 
labeled as a form of energy, is subject to energy conservation, but the colloquial term heat, referring ambiguously to thermal energy, a heightened temperature, or a sensation of warmth, may be "generated" in various everyday processes (such as rubbing). On the other hand, colloquial use of the word heat may be distracting for physicists. In what follows, we use both terms.

\section{PREVIOUS RESEARCH}

Canonically incorrect ideas are seen as taking different roles in the learning process, according to different researchers and different accounts. In one body of literature, canonically incorrect ideas are seen as obstacles to learning; in a different body of literature, canonically incorrect ideas are seen as having the potential to support intellectual progress, depending on the context. In our analysis of a series of episodes from a professional development course, we will argue that a particular, canonically incorrect idea-that gas particle collisions generate thermal energy-leads to a more canonically correct, more scientifically sophisticated idea in our particular instructional context. Before we present our analysis, we establish that the literature frames the collisions-generate-heat idea as a common, canonically incorrect idea, and we discuss the different roles that the literature ascribes to this and other canonically incorrect ideas.

\section{A. The idea that individual particle collisions generate thermal energy is a common canonically incorrect idea}

The idea that collisions among ideal gas particles generate heat has been identified as a common, canonically incorrect idea among university students and secondary teachers $[3,6-9]$. Students predict that adiabatic compression will increase the temperature of a gas because the gas particles will collide more often, producing more energy, heat, or friction. For example,

"More collisions between particles, more energy produced due to friction." [3]

"Same number of particles in smaller volume, then particles more squashed, more collisions, more heat produced." [3]

"[The molecules] start bumping into each other, causing friction. It's like rubbing my hands against each other, friction will increase temperature, because my hands get hotter." [6]

"Increasing the number of molecules per unit [volume] will increase the temperature, because they have an increased number of collisions and those collisions are expressed as heat." [6]

This kind of explanation contradicts the accepted disciplinary account of temperature increase during adiabatic compression, which is that kinetic energy transferred to the gas during compression dissipates into thermal energy. Rozier and Viennot interpret these responses as indicating that "macroscopic properties of bodies colliding inelastically are ascribed to microscopic particles" [3].

Robertson has observed similar explanations in scenarios involving adiabatic expansion:

"The volume is increased, giving the particles more room to move around so they collide less, causing the temperature to decrease." [9]

"The temperature decreases because increasing the volume cause[s] the number of collisions per second per unit area to decrease and the number of collisions between particles to decrease. This causes the particles to slow down because they are not colliding as often, resulting in a decreased temperature." [9]

One possible explanation for this association is the idea that collisions sustain the particles' kinetic energy (and thus the temperature of the gas). Another possible explanation is the idea that if collisions generate warmth, lowering the collision frequency would result in cooling.

Explanations indicating the idea that gas particle collisions generate thermal energy were given by about half of the 50 American university students interviewed [6] and $40 \%$ of the 2000 French university students responding to a written questionnaire [3]. Though only $10 \%$ of American students responding to a written task showed evidence of this idea [8], researchers suggest this may have only been because they did not explain their ideas fully; students in interviews who initially gave macroscopic arguments "very quickly introduced microscopic ideas to support their assertions when asked for further details." These researchers suspect that such reasoning may underlie many students' reasoning about ideal gases [3].

\section{B. Canonically incorrect ideas may be obstacles to learning in certain contexts}

Some researchers see canonically incorrect student ideas as obstacles to learning in that they may stand in the place of canonical ideas [10-12]. Students who are intellectually satisfied with canonically incorrect ideas may lack a motive to replace or reorganize such concepts [12]. The appropriate instructional approach, in this perspective, is to help learners confront their misconceptions early in the learning process, showing them that their ideas are in conflict with other ideas or with observed evidence $[12,13]$.

Several researchers suggest that the particular idea that collisions generate heat is an obstacle to learning $[3,6,7,9]$. This canonically incorrect idea has internal inconsistencies that are seen as undermining scientific reasoning, "allow[ing] comments which, in the accepted theory, lead to contradictions" [3]. For example, researchers 
assume that students who argue that "collisions generate heat" "do not realize that this mechanism does not allow for a steady state in which the temperature does not change" [7] and "are unaware (or unconcerned) that the process they proposed could not increase the temperature without violating the principle of energy conservation" [8]. Since the idea that collisions generate heat provides an account of the change in temperature of a gas under adiabatic compression, it may prevent students from seeking an alternative (canonically correct) mechanism; it may be more satisfying to students than a canonical alternative, causing students to reject the correct explanation. For example, Kautz argues that when students attribute a change in the internal energy of the gas "to a process inside the system rather than an interaction with its surroundings" (i.e., when they attribute the change in temperature to the increased collision frequency of the gas particles), they fail to recognize "the role of work and heat in transferring energy between a system and surroundings" [7]. In summary, the idea that gas particle collisions generate heat in processes such as adiabatic compression is an example of a common, canonically incorrect idea that researchers have understood as being a barrier to learning.

\section{Canonically incorrect ideas may be productive in certain contexts}

In this paper, we present examples of learners' canonically incorrect ideas being productive, rather than obstacles to learning, in a certain context. Researchers have used the term "productive" in a variety of senses, all with the general aim of recognizing that ideas that are not canonically correct can serve as valuable intellectual raw material [14-16] that learners use to make intellectual progress. Elby and Hammer define an idea as productive to the extent that it "generates behavior, attitudes, and habits that lead to 'progress' as defined by the given person or community" [17]. Engle and Conant, similarly, use productivity to refer to "getting somewhere" intellectually [18], whether closer to the canonically correct answer, or increasing the quality of an idea in some other sense. Our use of the term productive in this paper is consistent with both of these definitions: an idea is productive when it supports, initiates, or sustains progress. Harrer's perspective on a productive idea is less about what it leads to and more about its situated correctness: he defines ideas as productive if they are "correctly applied in context" [19]. All of these authors allow for ideas to be productive in a variety of senses, e.g., epistemologically, affectively, or conceptually.

In the discussions of adiabatic compression we describe in Sec. V, the idea that collisions generate heat plays an important role in the evolution and negotiation of the teachers' ideas, which ultimately land on an integrated, canonically correct model for how kinetic energy transforms into thermal energy in this scenario. We will argue that in this context, the idea that "collisions generate heat" is not an obstacle to learning: its presence does not inhibit the teachers from negotiating their understanding of their energy model and their commitment to mechanistic reasoning. In particular, it is not the case that teachers fail to recognize the role of heat or work in transferring energy to the gas, or ignore the inconsistency between the collisionsgenerate-heat idea and the steady state of the gas. Instead, the collisions-generate-heat idea is productive in the sense that it initiates intellectual progress. Specifically, the collisions-generate-heat idea initiates the reconciliation of the teachers' energy model with mechanistic reasoning about the phenomenon.

We expect that whether an idea is productive or is an obstacle to learning is influenced in part by the context in which the idea is used. In the context of short-answer written questions, in which the first idea to come to mind may be recorded with a sense of completing a task, an idea such as "collisions generate heat" may indeed inhibit further reasoning and thus be unproductive. In the context of an interview or classroom that emphasizes mechanistic reasoning and negotiation of developing concepts, an idea such as "collisions generate heat" may function as a starting point for extended scientific reasoning. In Sec. V, we demonstrate that the idea that collisions generate heat is productive in this latter sense. In Sec. VI, we identify the features of the instructional context that contribute to its productivity.

\section{RESEARCH CONTEXT}

\section{A. Instructional setting}

Our data consist of videotaped episodes of teachers analyzing the energy dynamics of specific real-life physical scenarios in the context of a professional development course. The episodes are taken from video records of professional development courses for secondary teachers offered through Seattle Pacific University as part of the Energy Project, a five-year, NSF-funded project to develop and study teacher practices of formative assessment in the context of energy teaching and learning. The course in which the events of Sec. V took place was held in the summer of 2013. Sixteen teachers participated, all of whom had taken another Energy Project professional development course in a previous summer. Middle school and high school teachers worked together in this course as intellectual equals, with no distinction made between these two subpopulations. The course makes extensive use of a suite of energy representations called Energy Tracking Representations, including a role-playing activity called Energy Theater and a graphic representation called an Energy Tracking Diagram. These are described in detail in Sec. IVA. The themes of the course included (1) mechanisms of energy 
transfer and transformation and (2) energy degradation [20]. The data for this paper are from the first week of instruction, which focused on mechanisms of energy transfer and transformation.

\section{B. Data collection and episode selection}

Energy-centered professional development courses offered by the Energy Project are documented with video, field notes, and artifact collection (including photographs of whiteboards, written assessments, and teacher reflections). In each course, teachers are grouped into 4-8 small groups of 3-4 participants each, and two groups are recorded daily. As researcher videographers document a particular course, they take real-time field notes in a cloudbased collaborative document, flagging moments of particular interest and noting questions that arise for them in the moment. Later, the researcher videographers or other members of the Energy Project identify video episodes to share with a research team. We use the term "episode" to refer to a video-recorded stretch of interaction that coheres in some manner that is meaningful to the participants [21]. These episodes are the basis for collaborative analysis, development of research themes, literature searches, and the generation of small or large research projects.

The episodes in this paper were selected from an Energy Theater enactment initially observed by author Scherr in the summer of 2013, who was an instructor at the time. In this enactment, participants negotiate, perform, and reflect on an Energy Theater for adiabatic compression of a gas. Scherr highlighted this particular Energy Theater enactment on the basis of audio-visual clarity and sustained learner engagement with a physical scenario, specifically, a scenario in which learners negotiated the mechanism for an energy transformation. Because identifying and labeling mechanisms for energy transfer and transformation were emphasized for the first time in the summer of 2013, we wanted to better understand how learners interacted with this new feature of our representations.

As we began to analyze learner engagement with this particular energy transformation, we realized that the role that "collisions generate heat" played in the negotiation of the energy dynamics of adiabatic compression was not an obstacle to these teachers' learning but was instead productive for their learning. That is, in the context of our course, the same idea played a different role in learning than was represented in the literature. The episodes we selected illustrate the evolution of the teachers' ideas and the role that this idea played in this evolution, highlighting the importance of context to the role of canonically incorrect ideas in learning.

We do not present evidence that the data below is representative: that is, we do not present evidence that other negotiations involving canonically incorrect ideas have the same features (though our experience suggests that some of them do). Rather, we put forward this enactment as a case of the productivity of a canonically incorrect idea, through which we may refine our sense that the role of canonically incorrect ideas depends on the instructional context.

\section{Analytic framework}

We take the theoretical perspective that the general properties of an event or phenomenon emerge from the specifics of a particular case, rather than from the patterns that emerge across cases [22]. Our methodology is to identify video episodes in which learners engage with energy concepts in general and conduct detailed analysis to characterize the specific concepts with which they engage [21]. Through ethnographic analysis of learners' embodied interactions with each other [23-26] and the material setting [21,27-29], we build plausible causal links between the idea that collisions generate heat and the teachers' subsequent development of a more correct account of energy transformation (meaning an account that integrates the energy model with mechanistic reasoning) [30-32]. This development is observed at the level of the group and is indicated by changes in various participants' verbal and behavioral interactions with one another, rather than being assessed for individual participants.

After identifying this enactment as one likely to enhance our understanding of learner engagement with mechanisms for energy transfer and transformation, each author watched the video multiple times, creating a detailed narrative of events as well as a transcript. On the basis of the narrative, transcript, and multiple viewings, the significance of these episodes emerged as contributing to theory about the role of canonically incorrect ideas in science learning. Claims were developed that connect our case to existing literature on learning theory and on the specific canonically incorrect idea that these learners initially enact. Four episodes from within the enactment (described in Sec. V) were isolated and transcribed to illustrate the productivity of a specific incorrect idea in these teachers' development of a more correct idea, as well as the importance of context for an idea's productivity.

\section{SCIENTIFIC PRACTICES FOR UNDERSTANDING ADIABATIC COMPRESSION}

Our professional development courses emphasize the development and use of (1) a strong conceptual model of energy - that is, a model of energy as conserved, localized, transferring among objects, and transforming among forms-and (2) a strong sense of mechanism-that is, accounts of how phenomena are caused by the activities of (possibly unseen) entities. The specific ideas and practices we emphasize are grounded both in our own sense of what 
is important for teachers to know, based on our experience as physicists and teacher educators, and in the content and practices highlighted by the Framework for K-12 Science Education [33] and the associated Next Generation Science Standards (NGSS) [34], to which teachers are accountable. The teachers featured in the episodes in Sec. V draw on these ideas and practices as they make sense of the adiabatic compression of an ideal gas. In this section we present the central characteristics of our conceptual model of energy and of mechanistic reasoning about energy, in preparation for our presentation of the evolution of the teachers' ideas in Sec. V. At each stage in this evolution, these ideas and practices shape the teachers' negotiation.

\section{A. Conceptual model of energy}

Conceptual models, as understood in the NGSS, are "explicit representations that are in some ways analogous to the phenomena they represent" [33,34]. Their purpose is for investigators (learners or scientists) to better visualize a phenomenon. Conceptual models may include diagrams, physical replicas, mathematical representations, or other explicit representations. In the context of the teacher professional development courses for this study, the conceptual model of energy includes a suite of representations called Energy Tracking Representations [35]. One of these is a role-playing activity called Energy Theater; another is a graphic representation called an Energy Tracking Diagram. Each of these representations supports analysis of energy transfers and transformations in complex real-world scenarios, such as a refrigerator cooling food or a light bulb glowing steadily. The rules of Energy Theater are as follows $[5,35]$ :

- Each person is a unit of energy in the scenario.

- Regions on the floor correspond to objects in the scenario.

- Each person has one form of energy at a time.

- Each person indicates his or her form of energy in some way, often with a hand sign.

- People move from one region to another as energy is transferred, and change hand sign as energy changes form.

- The number of people in a region or making a particular hand sign corresponds to the quantity of energy in a certain object or of a particular form, respectively.

The rules of Energy Tracking Diagrams are as follows [36]:

- Schematic areas on a whiteboard or on paper correspond to objects in the scenario.

- Individual units of energy are represented as individual letters, with the specific letter representing the form of energy.

- Energy transfers and transformations are represented with arrows. All arrows have a letter at the head and the tail. Arrows that cross the boundaries of object areas indicate energy transfers. Arrows that have a different letter at the head than the tail indicate energy transformations.

- The process by which a transfer or transformation occurs (e.g., mechanical work, metabolism, conduction) is indicated by the color or label of the arrow.

- Sequential order of energy transfers and transformations is represented by sequences of letters and arrows.

- Relative amounts of energy may be represented with coefficients on the letters that represent units of energy.

Energy Tracking Representations instantiate specific concepts about energy including conservation, localization, transfer, and transformation:

Conservation.- The principle of energy conservation is a foundation of the study of energy in physics. A primary advantage of the Energy Theater model, in which each participant is a unit of energy, is the requirement that participants enact a strong commitment to energy conservation: people are not created or destroyed. Energy Tracking Diagrams also explicitly represent energy conservation in that units of energy (letters) are explicitly visualized as persisting all the way through the represented scenario.

Localization. - Energy is localized in objects and fields. In Energy Theater and in Energy Tracking Diagrams, energy is located in objects, consistent with goals appropriate to secondary instruction. Kinetic energy, in particular, is located in bulk objects that have mass and speed; thermal energy is located in objects according to their temperature. In physics scenarios, ideal gases are usually in containers at rest, and so have no kinetic energy in the bulk sense except when the movement of a container wall causes many particles to move the same way at the same time. Thermal energy is modeled as the total kinetic energy of all the gas particles, moving in all directions (rather than all in the same direction), whose individual speeds are represented by the Maxwell-Boltzmann distribution. The NGSS reinforces the idea that energy is associated with either the motion or configuration of specific objects, stating that learners should know that "the energy of macroscopic objects can be understood in terms of the energies of the microscopic particles that comprise those objects" [33,34].

Transfer.- Physical phenomena frequently involve a transfer of energy from one object to another. The NGSS identifies energy transfer and flow as high priorities, e.g., "Students should trace where energy comes from and goes next in examples that involve several different forms of energy along the way" [33,34]. In Energy Theater, energy transfer is represented by participants moving from one object area to another; in Energy Tracking Diagrams, energy transfer is represented by arrows originating in 
one object area and ending in another. Kinetic energy transfers via work, thermal energy transfers via heating, and electromagnetic energy transfers via radiation. These are the only energy transfer processes, other than processes that also transfer matter (such as electrical conduction and thermal convection). In adiabatic compression, kinetic energy is transferred from the moving wall of the gas container to the individual gas particles by means of collisions in which the wall does work on the particles it impacts (and thereby on the gas as a whole).

Transformation.- Energy forms, in our model, are categories of evidence that energy is present or changing, and thus an important means of connecting a unified energy concept to a variety of observable phenomena [37]. The NGSS warns that the idea of forms can be "misleading, as it implies that the nature of the energy in [different] manifestations is distinct when in fact they are all ultimately [not distinct]" $[33,34]$. However, forms are useful for associating energy with its observable indicators, which the NGSS supports. Kinetic and thermal energy are both subtypes of motion energy and can transform into one another: for example, in adiabatic compression, kinetic energy transforms into thermal energy by means of dissipation.

\section{B. Mechanistic reasoning about energy}

Understanding the process by which kinetic energy in the wall becomes thermal energy in the gas in adiabatic compression requires teachers to reason mechanistically about the scenario. By mechanistic reasoning, we mean that teachers must not only understand that a particular cause leads to a particular effect but also how this particular cause brings about this particular effect [38-44]. Russ and coauthors [38,39] adopt the term "chaining" $[42,43]$ for describing how each stage of a mechanism "necessarily follows from the one before it and necessarily leads to the one after it," and consider chaining to be "strong evidence of mechanistic reasoning" [39]. In this perspective, the central components of mechanisms are activities (the "components of mechanisms that produce change") and entities (the things that engage in activities). For example, Russ and coauthors compare an explanation of the causal relationship between a pressure and volume change in terms of the ideal gas law (not necessarily mechanistic) and kinetic-molecular theory (mechanistic) [38]. Using the ideal gas law does not require chaining; one can simply note the inverse relationship between pressure and temperature, all else constant. Using the kinetic molecular theory, on the other hand, requires understanding how changing the volume of the gas affects the frequency and intensity of collisions between the gas particles and the wall of the container.

This sense of mechanism-as explaining the sequence of events by which $A$ causes (or becomes) $B$-is echoed by the NGSS. "Constructing explanations," including mechanistic explanations, is one of the central practices that the NGSS seeks to promote, and "Cause and effect: Mechanism and prediction" is one of the seven crosscutting concepts considered to be "fundamental to the nature of science" [33,34]. The NGSS's definition for mechanismas "a model for the chain of interactions that connect $A$ and $B$," where $A$ is a cause and $B$ is an effect-closely mirrors that described above.

Both because of its centrality to the content and practices of science, and because of its importance to the current vision of K-12 science education, mechanistic reasoning plays a significant role in our professional development courses for K-12 teachers. Although energy itself is "not a description of a mechanism" [45] in the sense that energy (unlike a force) does not cause things to happen, richly scientific depictions of the energy dynamics of a system or scenario include mechanisms for energy transfer and transformation. For example, representations of kinetic energy transfer between objects may include descriptions of a mechanism for this energy transfer, such as a collision in which mechanical work is done, and representations of the transformation of kinetic to thermal energy may identify the dissipative process by which this transformation occurs.

As we describe above, our model for professional development draws heavily on a set of Energy Tracking Representations. Teachers represent mechanisms for energy transfer and transformation by labeling (or coloring) the arrows in their Energy Tracking Diagrams with a specific mechanism, such as elastic compression, dissipation, metabolism, or conduction.

The teachers that appear in the episodes below are enacting Energy Theater and then drawing Energy Tracking Diagrams. Although there was no strict enforcement of declaring mechanisms for energy transfer and transformation during their enactment of Energy Theater, nor of labeling the arrows in their Energy Tracking Diagrams, they spontaneously use mechanistic reasoning to decide about and make sense of the transformation of kinetic to thermal energy during the adiabatic compression of an ideal gas. The canonical description of this phenomenon is that kinetic energy transforms into thermal energy via the mechanism of dissipation: Initially, the moving wall (pushed by a person) transfers kinetic energy to the gas particles closest to it via collisions between the wall and the gas, and the speed of these particles' bulk motion in the direction of the wall's movement increases. As these sped-up gas particles randomly interact with the remaining particles of the gas, kinetic energy is transferred among them, and the initial increase in bulk motion becomes an increase in the random motion of the gas particles. In this sense, the kinetic energy of the gas becomes thermal energy of the gas via the mechanism of dissipation. As we will see, the teachers in the episodes below gradually refine their initial ideas via the use of 
reasoning about mechanisms for energy transfer and transformation at the microscopic level.

\section{PROGRESSION OF TEACHERS' IDEAS ABOUT ENERGY TRANSFORMATION IN ADIABATIC COMPRESSION}

In what follows, we describe a series of ideas that teachers convey in their collaborative work to model energy transfers and transformations in adiabatic compression of a gas. An initial idea - that thermal energy is generated by individual collisions-stimulates two different responses: that thermal energy must come from somewhere (and a complete analysis must show where it comes from), and that thermal energy is a collective phenomenon, not associated with individual particles. Eventually, the teachers reconcile their ideas by recognizing that the thermal energy of the gas is dissipated kinetic energy. At each stage of this specific progression of ideas, teachers demonstrate their commitment to a strong sense of mechanism and a strong model of energy. These two commitments shape their negotiation of ideas and, ultimately, support them in reconciling competing conclusions that are generated.

The teachers in this course were not explicitly asked to "analyze adiabatic compression," i.e., they were not offered technical terms for the scenario they analyzed. Rather, they were shown the scenario by means of the PhET simulation [46] titled "Gas Properties," which features a tank of visible particles that may be heated, cooled, or compressed by means of various devices in the simulation (Fig. 1). The instructor demonstrated compression of the gas by quickly moving the left wall inward (in the simulation, this wall is pushed by a human figure). This action affects the movement of the gas particles in the simulation (those near the left wall are pushed inward and collide with other particles) as well as the temperature of the gas (it rises). When the wall stops moving, the temperature stabilizes. The instructor asked the teachers to analyze the scenario that she had demonstrated. According to the established routine of the class, each half of the class (eight teachers) first worked together to negotiate an Energy Theater representation of the scenario, then split into two groups of four to create Energy Tracking Diagrams for it.

The first episode we present occurs approximately 25 min into one-half of the class's negotiation of the Energy Theater for the adiabatic process represented in the simulation. Eight teachers have been participating in the small group discussion: June, Scott, Denise, Sally, Andy, Elaine, Dan, and Brent (pseudonyms). The teachers have placed large loops of rope on the floor to represent the man (that pushes the wall), the wall, and the gas (sometimes referred to in bulk, as "the gas," other times referred to as "gas particles" or "purple balls," as depicted in Fig. 1). They have agreed that kinetic energy is transferred from the man to the wall, and are debating the transformation of kinetic to thermal energy; asking whether this is a

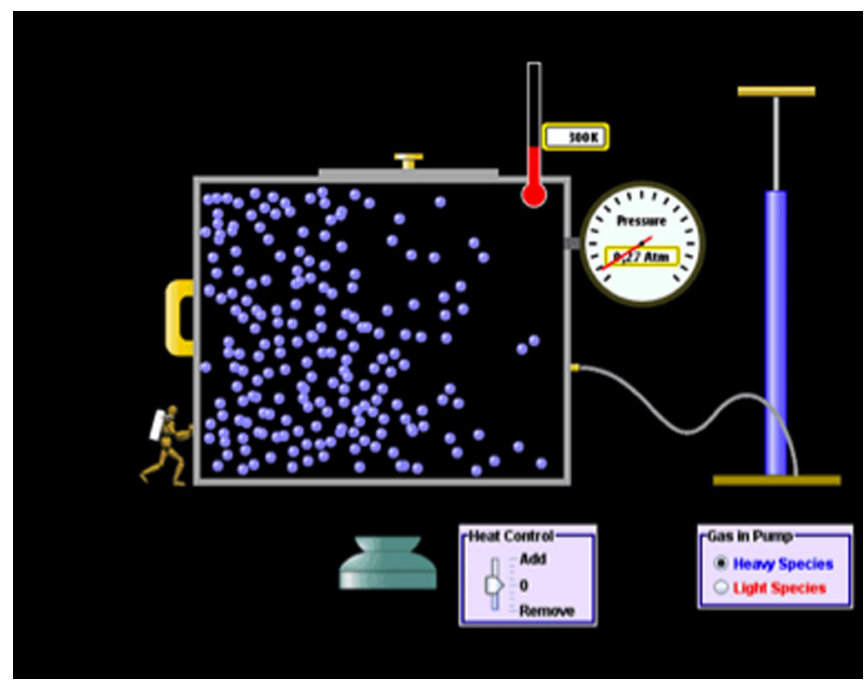

FIG. 1. Screenshot from "Gas Properties," a PhET Interactive Simulation [46].

transformation that is internal to the gas, whether kinetic energy turns to thermal energy at all, or whether thermal energy is just kinetic energy; and debating the mechanism by which kinetic energy transforms to thermal energy.

\section{A. Initial idea: Thermal energy is generated by individual collisions}

Approximately $25 \mathrm{~min}$ into this group's negotiation of the "energy story," June proposes a mechanistic account that is consistent with an increase in the temperature of the gas. She argues that heat is lost when the gas particles collide with one another (similar to the collisions-generateheat idea described in Sec. II A). When the gas is in its compressed state, the distance between the particles decreases, and June wonders whether the collisions become more "efficient" in transferring kinetic energy, thereby losing or wasting less heat (Video 1):

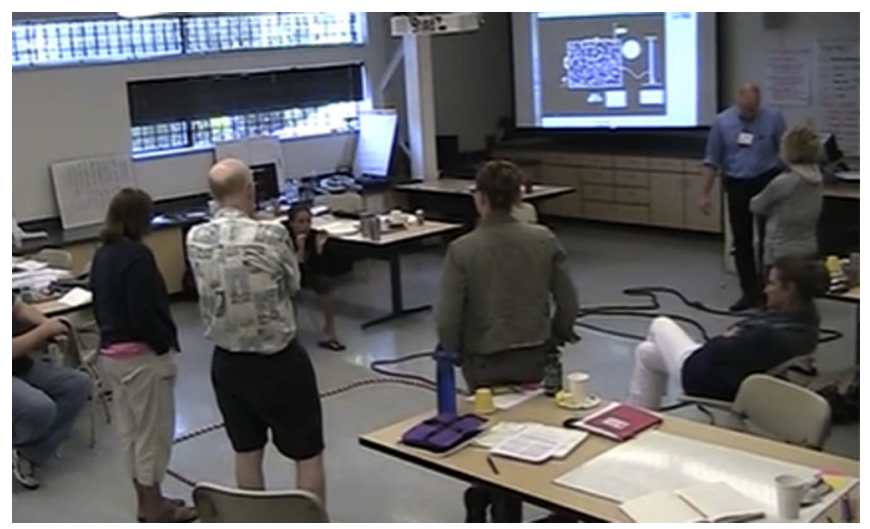

VIDEO 1. Participants prepare to enact Energy Theater for adiabatic compression of an ideal gas. In this episode, one participant argues that heat is lost when gas particles collide with one another. 
June: Is, does the transfer, does-as the volume decreases does the transfer of, whatever we're going to call that energy between particle to particle become more efficient? So,

Scott: Huh?

June: Because we say that particle to particle there's a transfer, something's happening in this transfer, as that space between particles decreases, does the transfer become less wasteful, and therefore harnessed? [inaudible] temperature? Anybody see what I'm saying?

Rachel (course instructor): So I want to hear a couple things, there are a couple of things that you're saying: you're wanting to talk about collisions between the particles, and you're thinking there is a loss.

June: We've talked about that. We've talked in our group about, every transfer every transformation there's a heat loss.

Scott: Oh, yeah.

June: So now we're decreasing the amount of lost heat, and making it more effective.

Sally: Thermal energy. Are you saying that there's thermal energy lost at every transfer?

June: I don't know the difference. I don't even know.

Scott: Right.

Rachel: You're saying that there's thermal energy lost, when an individual particle collides with another individual particle there's thermal energy lost in that collision.

June: Yes.

Scott: Yeah.

Rachel: So that's different than what I heard somebody else saying which is that a single particle doesn't really have thermal energy, it just has kinetic energy. Just plain old velocity.

June: But the, but the transfer. The transfer or the transformation that we talked about with our systems, is the byproduct, heat, thermal energy, whatever we're calling it, is the byproduct of a transfer or transformation that has occurred.

Here, June proposes a mechanism for the energy transfers and transformations she has modeled. She seeks to account for the observation (in the simulation) that the temperature of the gas increases, corresponding to an increase in the thermal energy of the gas. She posits a mechanism by which the kinetic energy of the gas particles becomes the thermal energy of the gas, and she attributes this transformation to the collisions of the particles. This particular mechanism instantiates many of the features of mechanistic reasoning advanced by Russ and colleagues [38,39]: June identifies the relevant entities (the particles of the gas) and activities (particle-particle collisions) and links them with causal reasoning via chaining (heat is lost during particle-particle collisions; these collisions are thought to be more efficient when the gas is compressed; therefore, less heat is lost in the compressed state of the gas).

At least one link in June's chain of reasoning is tenuous: she does not explain how "decreasing the amount of lost heat" leads to an increase in temperature. (It seems that "less heat lost" by the particles would instead lead to a smaller decrease in the temperature of the gas.) However, June does not explicitly claim that this mechanism leads to an increase in temperature.

In what follows, we will show that the idea that collisions generate heat is productive in this instructional context: it serves an important role in the evolution of the teachers' ideas, which ultimately land on an integrated, canonically correct model for how kinetic energy transforms into thermal energy in this scenario. The mechanistic reasoning that June initiates in this episode sets teachers up to iteratively negotiate the connection between (i) mechanistic reasoning about the transformation of kinetic to thermal energy and (ii) a coherent model for the energy of the gas. As we will see in the following episodes, through June's probing questions about the role of collisions-and through the mechanisms she attributes to these collisions - she invites Andy, Denise, and Sally to articulate counterarguments (based on their model of energy) and alternative mechanisms for energy transformation. Thus, both the mechanism June proposes here and her sustained commitment to mechanistic reasoning are productive.

\section{B. Response: The thermal energy must come from somewhere}

Shortly after June proposes the mechanism that collisions generate thermal energy, Sally points out an inconsistency between this mechanism and her own model for energy. She argues that the thermal energy units (" $T$ ") that are "produced" in this scenario must "come from" somewhere, and the salient candidate for her is that it "comes from" the particles-that the motion energy (" $K$," for kinetic) of the particles becomes thermal energy. If this is the case, she says, the particles would slow down and, as a result, the temperature would go down. However, this is not what the simulation represents (Video 2):

Sally: If there is thermal energy that is being produced from the motion of the collisions and all of that that we are saying, I don't know if I agree with this statement.

Elaine: Right.

Sally: I don't know what I know right now.

Elaine: We know something.

Sally: Does that mean-Where is that heat energy coming from? It's coming from, what I'm hearing, the motion. Right?

June: I think it's... 


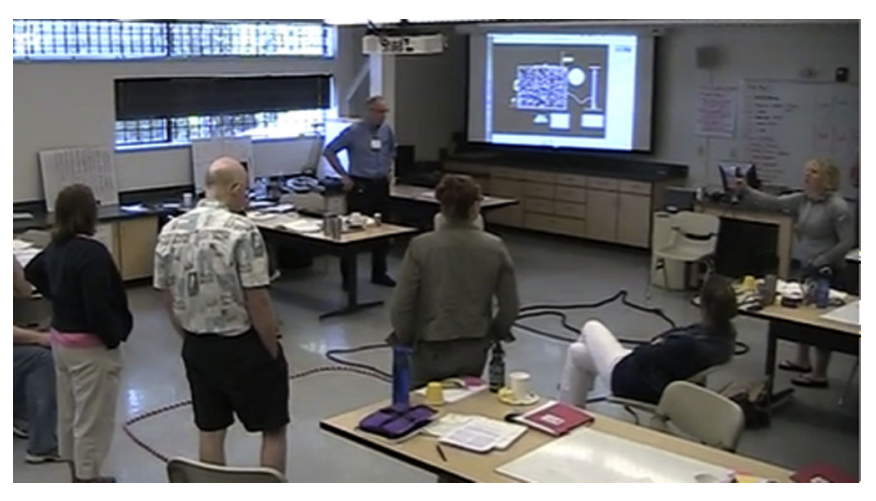

VIDEO 2. Participants prepare to enact Energy Theater for adiabatic compression of an ideal gas. In this episode, one participant points out an inconsistency between the collisionsgenerate-heat idea and her own model for energy.

Sally: Okay, the collisions. Heat energy is being produced from that. Right? So are we saying that those balls will eventually slow down?

Scott: Yeah. If there was internal friction within the balls, that you had some loss due to, instead of...

Sally: Let's, it's a closed system. Are those balls just going to continue [getting] around like that? And we can see that on the diagram because the temperature's not going down.

Scott: Right.

Elaine: No.

Sally: So, is there $K$ to $T, T$ to $K, K$ to $T, T$ to $K$, or, is there no $T$ there in the first place because it's all kinetic? I have no idea.

Elaine: What's wrong with $K$ to $T, T$ to $K$ ? $K$ to $T$ slows it down, but then the heat en, the thermal energy back to kinetic speeds it up again.

Sally: I'm just saying is it a back and forth, back and forth transformation. [Inaudible] equilibrium.

Scott: I have a problem with that one.

Here, Sally is actively seeking a mechanism that is consistent with her model for energy. She challenges June's mechanism on the grounds that it is inconsistent with her model for energy: if the collisions were generating thermal energy, that thermal energy would have to come from somewhere, but if it comes from the particles, they must be slowing down (whereas, according to the simulation, the particles have constant average speed). Scott agrees with Sally's challenge, attributing a different mechanism than June to her own argument-that it is the friction between the particles during the collision that generates heat.

Sally suggests one way of reconciling the idea that collisions generate thermal energy with the observation that the gas particles do not slow down, imagining a continuous back and forth between kinetic and thermal energy: $K$ to $T$, $T$ to $K, K$ to $T, T$ to $K$. She questions this, wondering about the distinction between kinetic and thermal energy at the microscopic scale: "is there no $T$ there in the first place because it's all kinetic?"
Contrasting the account in some research literature $[3,7,8]$, in this case Sally and Scott recognize that this particular mechanism - that collisions generate heat-is inconsistent with the steady state of the gas, and recognize the role of heat or work in transferring energy to the gas. Not only does the presence of the idea that "collisions generate heat" not exclude these other understandings, the idea seems to stimulate negotiation of model-based and mechanistic reasoning.

\section{Response: Thermal energy is a collective phenomenon}

About 15 min later, after the group of eight teachers has negotiated and performed the Energy Theater for the "macroscopic scenario" (i.e., for the gas as a whole), the group splits into two small teams, each charged with working out the Energy Theater for the "microscopic scenario" (in terms of the particles constituting the gas). This episode begins as five of the teachers-Andy, Denise, June, Sally, and Elaine- - begin to draw an Energy Tracking Diagram for this microscopic process. As they do so, Elaine asserts that kinetic energy transforms to thermal energy in individual particle collisions. Andy counters Elaine's statement (Video 3):

Elaine: So some of these $K$ 's [pointing to units of kinetic energy on the diagram] when they collide will go to $T$, and some of these $K$ 's [pointing to diagram] when they collide will go to $T$.

Andy: I don't think so.

Elaine: Because isn't there always...

Andy: We do say, like if you have two cars, and they have a collision, then there's going to be heat produced. But that's because we're looking at large macroscale. I think when you're looking at the microscale with just the particles, I think $T$ is not a super helpful model. Thermal

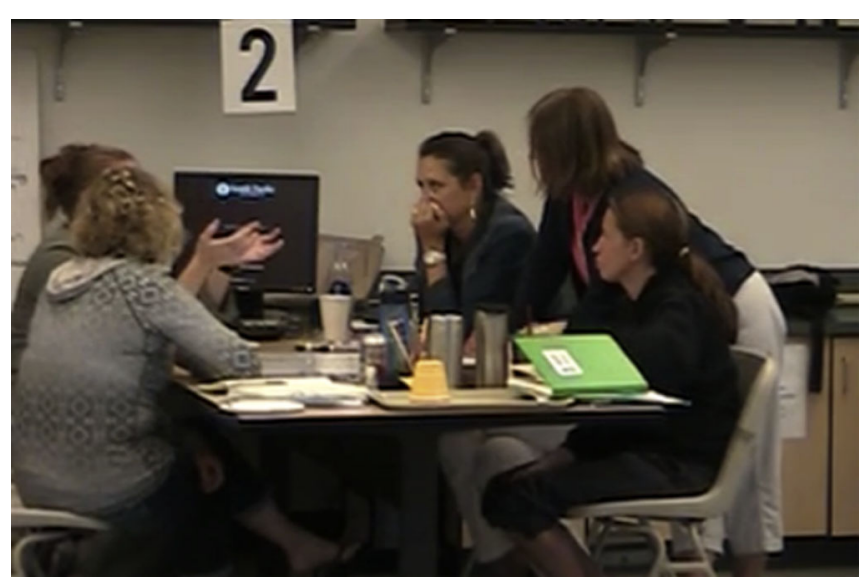

VIDEO 3. Participants construct an Energy Tracking Diagram for adiabatic compression of an ideal gas. In this episode, participants establish that thermal energy is a collective phenomenon. 
energy. Specifically because, when Stamatis [course instructor] was talking about the wheel? You know when he had the wheel arm with each different segment had kinetic energy? I think this is our situation here where each particle has kinetic energy. But then when you have rotation as a whole,

June: The sum has changed.

Andy: it's the sum, and that's your rotation. So then when we have...

Elaine: Okay then where does thermal come from?

Andy: I think it's the whole box of particles, you add up all the kinetic energy,

Elaine: So you don't have thermal, here.

Andy: Right. You've only got it when you're looking at the whole bunch of them together.

Sally: The bulk. Is when thermal comes in.

Andy's response to Elaine's idea distinguishes microscopic collisions from macroscopic ones, and then draws on an instructional example from the prior afternoon (relating kinetic and rotational energies) to assert that thermal energy is a collective phenomenon, not associated with individual gas particles. The function of Andy's explanation is to refute the proposed collisions-generateheat mechanism on the basis of her energy model. In other words, Elaine's statement of a canonically incorrect idea prompted Andy to establish an important component of the teachers' developing model of thermal energy. Andy does not propose an alternate energy transformation mechanism during this interaction.

\section{Reconciliation: Thermal energy is dissipated kinetic energy}

In the next ten minutes, Andy, Denise, June, Sally, and Elaine collaboratively construct an Energy Tracking Diagram for the microscopic scenario. They discuss the difference between thermal energy and temperature: they agree that a larger object at the same temperature will have more thermal energy, and decide that they would represent this on their Energy Tracking Diagram by drawing more $T$ 's (thermal energy units) in the larger object. They then wonder together how they might represent the change in the gas tank's volume on their Energy Tracking Diagrams. June states that she does not know why decreasing the volume causes the temperature to rise. Denise proposes that decreasing the volume will increase the frequency of collisions with the container wall, "increasing the movement" of the gas particles and so increasing the temperature of the gas. Andy and Rachel (the course instructor) counter Denise's proposal, arguing that the energy of the gas particles is conserved when they collide, and that if hitting the walls increased the temperature, they would see the temperature increasing during the steady state of the gas. Rachel goes on to ask the teachers when, in the simulation, the gas heats up, and presses the teachers to identify how the inward motion of the wall affects the energy of the particles (Video 4):

Rachel (course instructor): So when does it heat up?

Elaine: When does it what?

Rachel: When does it heat up?

Sally: When it's that initial movement.

June: When the volume changes.

Rachel: I mean duh, when the wall moves. [pushing gesture]

Andy: Right.

Elaine: So there's a one way.

Rachel: [pushing gesture] So why does a moving wall do something to change,

Denise: And then it equalizes.

Rachel: I mean that's a dumb question, [repeating her own question in a singsong voice] 'what's different about the wall when it's moving?', well, it's moving! But, like, why does the moving affect it differently than just sitting there.

Andy: Cause now you've got...

Sally: Kinetic.

Andy: ... now you've got a ball coming out and a baseball bat hitting it [gestures fast hit], it's not just the ball bouncing [gestures rhythmic symmetrical oscillation].

Rachel: It's like I could stand here with my baseball bat [stands in bunting stance] and a baseball could come in and it would bounce off. But if I'm swinging the baseball bat [mimes swinging]

Denise: Right, so when it's...

Sally: You're adding an additional motion [sweeps open hand right to left]. So it's like the vroom

Rachel: Yes.

June: Why wouldn't you see a, zoop [gestures a vertical spike]?

Denise: Well you see an increase but then it stabilizes, that's the part that I wasn't...

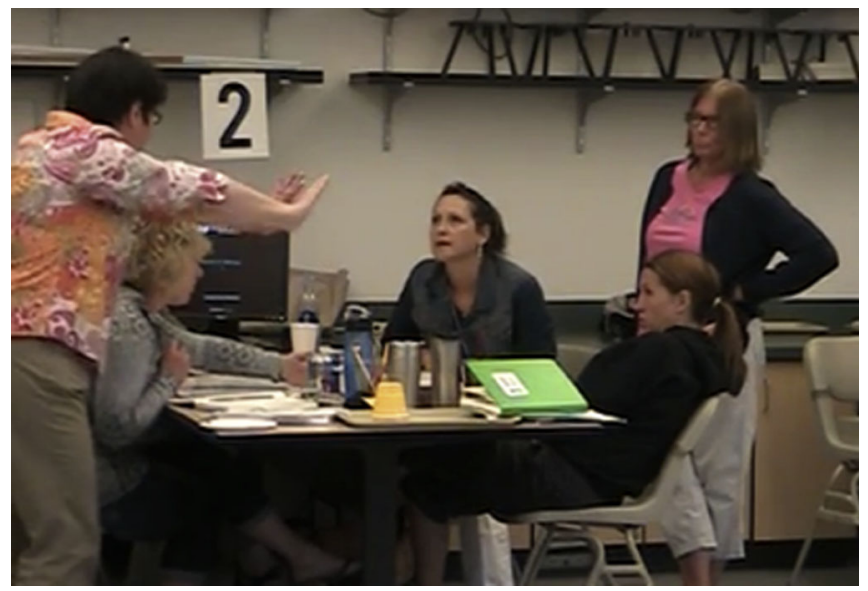

VIDEO 4. Participants construct an Energy Tracking Diagram for adiabatic compression of an ideal gas. In this episode, participants identify thermal energy as dissipated kinetic energy. 
Andy: Remember we saw that wave of particles, so those were the particles that hit the wall first, they were closest to the wall, the wall hits them [smack gesture], and then it evens out after all of our collisions distribute our energy [mixing gesture].

June: No, I, I, I hear what you're saying.

June, Sally, and Andy agree that the gas heats up when the wall moves inward, but do not initially offer a mechanism to account for the heating. When pressed, Andy makes an analogy to an incoming baseball that is hit by a baseball bat, emphasizing that hitting a ball with a bat is "not just the ball bouncing" (off the bat), and illustrating the asymmetry with gestures. Rachel revoices Andy's idea by contrasting bunting with swinging a bat. June wonders why this wouldn't produce a spike in the energy, rather than an increase that is sustained thereafter. In response, Andy explains that the transformation of kinetic energy to thermal energy is a distribution of that energy throughout the gas.

Andy's proposal signifies the teachers' successful reconciliation of their energy model with a mechanistic explanation of the temperature increase of the gas. Andy envisions kinetic energy being transferred from the moving wall of the gas container to the individual gas particles by means of collisions in which the wall does work on the particles it impacts: in Sally's words, "you're adding an additional motion." The entities in this mechanistic explanation are the wall and the gas particles (rather than only the gas particles, as in June's mechanistic explanation). The key activity is the wall hitting some of the particles (rather than particle-particle collisions). Andy's causal reasoning completes the mechanistic account: when the wall hits the particles, kinetic energy transfers from the wall to the particles. This account is consistent with the teachers' model of energy, in which energy is always conserved, and kinetic energy may transfer from one object to another in a collision.

Andy also offers a partial mechanism by which the kinetic energy in the gas particles hit by the wall becomes the thermal energy of the gas as a whole: she says that it "evens out after all of our collisions distribute our energy." Andy does not state here that the energy thereby becomes thermal energy, but this inference seems plausible given her earlier explanation of the collective nature of thermal energy (Sec. V C). In this explanation, the relevant entities are gas particles and the activities are particle-particle collisions, just as in June's original model. However, in Andy's explanation, the chain of events is that particleparticle collisions cause a series of kinetic energy transfers that ultimately "distribute" the kinetic energy throughout the gas (rather than generating heat that warms the gas). Andy's account is not only mechanistic, but also apparently consistent with the teachers' energy model.

The dissipated-kinetic-energy idea, represented most explicitly by Andy, appears in the classroom discourse as part of an extended response to the collisions-generateheat idea, represented most explicitly by June. The collisions-generate-heat idea is productive in the sense that it prompts the reconciliation of model-based and mechanistic reasoning about energy, initiating progress toward a more correct and scientifically sophisticated idea. June's idea prompts Sally to make sense of the implications of collisions-generate-heat for the motion of the gas particles; Elaine's take-up of this idea prompts Andy to articulate the meaning of thermal energy at the microscopic scale; and Denise's trying on a similar idea-that collisions increase the movement of the gas particles-prompts Rachel to draw the teachers' attention to the role of the wall in this scenario. This final step in the sequence of reconciliatory steps is the basis for Andy's successful reconciliation of the energy model with a mechanistic explanation for the increase in temperature of the gas. Andy's proposal is not only a reaction to June's idea; it is a refinement of it, involving some of the same entities (particles) and activities (collisions) and more complete chaining. In this sense, the collisions-generate-heat idea functions as an intellectual resource: it is the conceptual raw material that is refined into the intellectual product (the dissipated-kinetic-energy idea).

\section{E. Reflective discussion}

The teachers' discussions of the transformation of kinetic to thermal energy in the context of adiabatic compression continues for 40 more minutes, until the class takes a break for lunch. For the ten minutes following the episode presented in Sec. VD, the teachers continue discussing the adiabatic compression scenario within their small groups. Almost immediately after Andy proposes that the gas particle collisions distribute the kinetic energy transferred from the wall, Elaine leaves to rejoin her small group, and Rachel (the instructor) moves on to a different table. Andy, Denise, June, and Sally revisit their Energy Tracking Diagram and decide that it adequately expresses the scenario at the microscopic level. They discuss their open questions, many of which center around the relationship between temperature and kinetic energy; they seem to be coming to terms with the idea that temperature is a measure of the average kinetic energy of the gas, so more $K$ 's (kinetic energy units) on their microscopic diagram indicate that the temperature or thermal energy has increased. They briefly discuss the role of the wall in increasing the kinetic energy of the gas: they conclude that what matters for adding energy to the gas is not so much the change in the volume of the gas as the specific process by which the volume is changed.

After an exchange with Rachel about how they are representing collisions between particles on their Energy Tracking Diagram, the teachers assemble as a whole class for a reflective discussion. Rachel proposes that the class is now in a position to answer a question posted at the back of 
the room (among other open questions raised by the class): "By what means does $K$ go to $T$ ? And what does that have to do with warmth?" Brent draws a parallel to an earlier discussion in which the class discussed the relationship between kinetic energy and rotational kinetic energy: when a water wheel turns, the individual parts of the wheel have kinetic energy, but the whole wheel is thought to have rotational energy. Likewise, individual gas particles have kinetic energy, but the gas as a whole has thermal energy. When the kinetic energy of the particles is changed by the wall, it changes the thermal energy of the gas. In saying these things, Brent does not propose a mechanism by which $K$ goes to $T$, but he does connect a change in $K$ to a change in $T$. Rachel asks if other teachers have anything to add, and Akil highlights the difference between kinetic and thermal energy: One is associated with ordered motion and the other with disordered motion.

Rachel then asks June if she got her question answered as to whether or not there is a loss of thermal energy during a collision between two gas particles. June recaps her argument but then says she "doesn't think it matters for her." Andy and Scott offer rebuttals to the collisions-generateheat argument: that the temperature of the gas does not go down, and that the particles continue to move (i.e., they do not slow down). Stamatis (another course instructor) asks for evidence from their everyday experiences that particles do not slow down. Scott says that the atmosphere would settle, saying, "it would turn into a soup at our feet," and Sally proposes that water in a vacuum flask remains hot. At this point, the teachers return to their tables to write about this scenario in their journals.

In this large-group discussion, the teachers are both disambiguating kinetic and thermal energy and articulating how the collisions-generate-heat mechanism is inconsistent with their model for energy and their observations, both of the simulation and in their everyday lives. They do not explicitly state a mechanism for the transformation of kinetic to thermal energy in this wrap-up. Nonetheless, this reflective discussion represents a substantially different understanding of energy than when the scenario was first introduced: In those earlier conversations (see Secs. V. A and V.B), the teachers were not clear that the movement of the wall was consequential to the energy dynamics, questioned whether thermal energy was kinetic energy, and debated whether the macroscopic and microscopic Energy Tracking Diagrams told the same story. Their talk and action evolved toward a more complete and mechanistic account of the scenario, and the idea that "collisions generate heat" played a productive role in this evolution.

In the following days of the course, the teachers went on to analyze transformations of thermal energy to kinetic energy (e.g., in adiabatic expansion); recognize that some kinetic-to-thermal transformations are reversible (e.g., compression) but others are not (e.g., rubbing, deformation); realize that even when the energy of two similar systems is the same the transferability of the energy can be different; identify the conditions that determine energy transferability; and negotiate a working definition of free energy [20]. Their analysis of the energy dynamics of adiabatic compression was the basis for extended development of sophisticated concepts in thermodynamics.

\section{RELATIONSHIP BETWEEN INSTRUCTIONAL CONTEXT AND PRODUCTIVITY OF "COLLISIONS GENERATE HEAT"}

We make two claims in this paper: (1) The idea that collisions generate heat is productive in this instructional context, and (2) the idea is productive, in part, because of the instructional context. We addressed the first claim in Sec. V by showing that the collisions-generate-heat idea initiates the reconciliation of model-based and mechanistic reasoning and that the teachers ultimately land on an integrated model for the energy dynamics of adiabatic compression. We address the second claim in this section by positing elements of our instructional context that influence the productivity of the collisions-generate-heat idea. In so doing, we stipulate that the same idea may not be productive in another instructional context. For example, in the context of written exams, the idea that "collisions generate heat" may be part of what causes students to answer incorrectly.

A primary feature of our instructional context is the explicit instructional goal of modeling mechanisms of energy transfer and transformation. In a course with this instructional goal, the collisions-generate-heat idea is a useful starting point for reconciling model-based and mechanistic reasoning. For example, this instructional goal may have directed June's attention to proposing a mechanism for the increased temperature of the gas. The same goal may have supported Sally's commitment to finding a mechanism consistent with her energy model and Andy's eventual reconciliation of model-based and mechanistic reasoning.

This explicit instructional goal is embedded in a culture of collaborative sense making, in which sufficient time is allotted for discussion. In this particular instantiation of collaborative sense making, the teachers try on one another's ideas in order to challenge and extend them: this is how June's original idea connects to Sally's insistence that thermal energy must come from somewhere, how Elaine's instantiation of "collisions generate heat" connects to Andy's assertion that thermal energy is a collective phenomenon, and how Denise's idea is refined by Rachel's question about the role of the wall. This kind of collaborative sense making takes time (about two hours in this case). The allotment of time also signals and reinforces a culture of sense making.

Our instructional context uses a suite of representations that embed and support the goal of modeling mechanisms of energy transfer and transformation and the culture of collaborative sense making. Energy Theater is an embodied 
learning activity that supports teams of teachers in theorizing mechanisms of energy transfer and transformation via embodied interaction in a material environment [5]. Energy Tracking Diagrams-diagrams that represent an Energy Theater enactment on paper-may support learners in identifying mechanisms of energy transformations even more strongly than Energy Theater does, since Energy Tracking Diagrams include transfer and transformation mechanisms explicitly. Both of these representations support and sustain the course's instructional goal and culture of collaborative sense making, which, in turn, influence the productivity of the collisions-generate-heat idea.

Finally, the use of the "Gas Properties" PhET simulation to represent adiabatic compression draws attention to both (a) the phenomenon itself (temperature increase) and (b) the entities and activities that comprise the mechanism for the phenomenon (including the gas particles and the movement of the wall). This representation of the scenario may have initiated or augmented the teachers' attention to reconciling model-based and mechanistic reasoning.

In short, the sense in which "collisions generate heat" is productive in this particular negotiation of ideas-that it initiates a sequence of reconciling model-based and mechanistic reasoning - is tied to particular elements of our instructional context. These elements may function to support the initiation itself and/or to sustain and augment the process of reconciliation. The instructional context is not the only reason the collisions-generate-heat idea is productive: the idea also has elements of a correct mechanism (entities and activities), providing useful raw material that the teachers refine into the dissipated-kinetic-energy idea.

\section{DISCUSSION}

In this section we address some possible counterarguments and offer potential instructional implications.

\section{A. Addressing counterarguments}

Some readers may question our assertion that the collisions-generate-heat idea is productive for this group of teachers on the grounds that we have not shown that individual teachers have made progress in their understanding of the energy dynamics of adiabatic compression. Our claim is at the group level: learning is indicated by changes in participants' collective verbal and behavioral interactions with one another, rather than at the level of individual participants' understanding. We provide evidence that the teachers' shared talk and action evolved toward an account of adiabatic compression that is more aligned with that of disciplinary experts, and it is in this sense we argue that the teachers learned. We do not provide evidence as to whether individual teachers acquired knowledge of the energy transfers and transformations involved in adiabatic compression; this evidence would be required if we were making a claim about individual participants' learning rather than about the shared intellectual progress of the group. Nor do we offer evidence as to whether the learning that occurred was stable; in fact, we do not necessarily expect learners to make smooth, linear progress toward expertise, but instead expect them to test out words and practices, often using terms and behaviors before they have fully internalized their meaning $[23,26,47]$. In short, the concern that individual teachers have not learned about energy in these four episodes comes from a different theoretical stance than the one we take up in our analysis.

A second concern may be about the generalizability of our results: one may be concerned that this series of episodes is too idiosyncratic to inform theory about the role of canonically incorrect ideas in learning. One may argue that in most cases, an incorrect idea will not be productive for learning and instead will act as an obstacle. This argument is bolstered by the prevalence of literature on misconceptions and student difficulties in science. However, a variety of researchers in a range of instructional contexts have documented the productivity of various canonically incorrect ideas [13,38,48,49]. For example, Gupta, Elby, and Conlin describe the productive role that a "misontology" for gravity - "thinking of gravity as "stufflike' "- plays in teachers' discourse around the question of why heavy and light objects fall at the same rate [13]. In their analysis, one teacher, Lynn, proposes that all masses can be broken into equally sized pieces and suggests that gravity may have the same pull on each, "effectively map[ping] gravity onto the force per unit mass, which would be the correct definition for gravitational field." Lynn's colleague Daniel continually challenges Lynn's reasoning, treating gravity as an addable quantity: twice the mass should equal twice the pull. Eventually, the class as a whole comes to consensus on the idea of inertia: that an object with eight times the mass would experience eight times the pull but be "[eight] more times not willing to move." Gupta, Elby, and Conlin argue that Lynn's argument - an instance of Galilean reasoningemerged from the attribution of substancelike qualities to gravity, and that these ideas fed into the correct "Newtonian compensation argument" [13].

Furthermore, within our own instructional context, we find many examples of ideas that are both canonically incorrect (or undeveloped) and productive [20,37,50,51]. Our informal sense is that it is not rare for such ideas to play a productive role in teachers' learning, moving them (and us) forward in understanding energy concepts. For example, Daane, Vokos, and Scherr found that teachers' sense of energy as being "used up" was not inconsistent with teachers' commitment to energy conservation; instead, it expressed an intuitive understanding of energy degradation [20]. On this basis, Daane, Vokos, and Scherr articulate learning goals for energy degradation and the second law of thermodynamics that incorporate teachers' intuitive ideas about energy, the relevant physics content being discussed, and the Next Generation Science Standards [34]. 
These examples from our own local context and from the literature provide additional support for the argument that canonically incorrect ideas can be productive for learning. These examples further illustrate our point that the role that canonically incorrect ideas play in learning depends on the context. Both our local courses and several of the contexts described in the papers we cite above emphasize sense making and focus on the development of mechanistic reasoning, whereas much of the literature that reports canonically incorrect ideas as obstacles to learning is conducted in contexts in which students are asked to provide reasoning for their answers to conceptual questions. In these latter cases, students' canonically incorrect ideas may in fact stand in the place of or inhibit correct reasoning and thus act as obstacles to their providing a correct answer.

\section{B. Summary and instructional implications}

It is common and reasonable for educators to be concerned about the correctness of learners' ideas. We care about our students and want to do right by them. If we let them persist in what seems to us to be incorrect thinking, we may worry that we are betraying their trust, or shirking our duty to hold students accountable. Consistent with this concern, we may design instructional experiences with the goal of exposing and repairing students' incorrect ideas $[12,13]$.

In contrast, our own experience as instructors is that learners' ideas always have some seed of correctness, and that attention to learners' productive ideas is among the most powerful tools for facilitating growth [52-54]. We find that, in practice, attending to learners' ideas requires active engagement by both instructors and peers and stimulates learners' own resources for sense making and problem solving [49,55-58]. We design instruction with the goal of eliciting and cultivating learners' productive intuitions. We aim for instructional environments that display the disciplinary substance of learners' ideas, adapting and discovering instructional objectives in response to learner thinking [20,59]. In this perspective, instructors need not address incorrect ideas in order to clear the path for learning. Rather, instructors expect to support learners in refining, organizing, and otherwise improving the scientific quality of their own ideas through application of scientific practices. One result of this approach is that each course has a unique trajectory that emerges from the interaction of learners' agency with instructors' judgment of what is worth pursuing $[49,55]$.

Our analysis documents secondary physics teachers negotiating a common, canonically incorrect idea about the production of thermal energy in adiabatic compression: that collisions between the particles generate heat. Canonically incorrect ideas such as this one have been characterized in science education literature as obstacles to learning. Our analysis demonstrates that, at least in this case, the collisions-generate-heat idea is not an obstacle, but rather plays a productive role in the construction of sophisticated thermodynamic accounts of phenomena. We attribute the observed productivity of the idea partly to the learning environment, which emphasizes mechanistic reasoning about energy transfer and transformation processes and collaborative sense making. On this basis, we suggest that the productivity of an idea is not only a property of the idea itself, but also depends on features of the instructional context. We take this finding as evidence that the structure and culture of an instructional environment has the potential to transform the role that canonically incorrect ideas play in teaching and learning. Instructors who wish to promote both student agency and student conceptual progress may wish to frame canonically incorrect ideas as potentially productive and to emphasize negotiation and reconciliation as a context for these ideas' evolution.

\section{ACKNOWLEDGMENTS}

We gratefully acknowledge all the elementary and secondary teachers who have participated in Energy Project courses for their generosity in making their reasoning accessible to the Energy Project team. We are grateful to Seattle Pacific University's Physics Education Research Group, including A. R. Daane, K. Gray, S. B. McKagan, L. S. DeWater, L. Seeley, and S. Vokos for substantive discussions of this work. This material is based upon work supported by the National Science Foundation under Grants No. 0822342 and No. 1222732.
[1] M. Wiser and S. Carey, When heat and temperature were one, in Mental Models, edited by D. Genter and A. Stevens (Erlbaum, Hillsdale, NJ, 1983), p. 267-297.

[2] P. A. Kraus and S. Vokos, The role of language in the teaching of energy: The case of heat energy, Wash. State Teach. Assoc. J. (2011).

[3] S. Rozier and L. Viennot, Students' reasonings in thermodynamics, Int. J. Sci. Educ. 13, 159 (1991).
[4] J. W. Warren, The teaching of the concept of heat, Phys. Educ. 7, 41 (1972).

[5] R. E. Scherr, H. G. Close, E. W. Close, V. J. Flood, S. B. McKagan, A. D. Robertson, L. Seeley, M. C. Wittmann, and Stamatis Vokos, Negotiating energy dynamics through embodied action in a materially structured environment, Phys. Rev. ST Phys. Educ. Res. 9, 020105 (2013). 
[6] C. H. Kautz, Identifying and addressing student difficulties with the ideal gas law, Ph.D., University of Washington, 1999.

[7] C. H. Kautz, P. R. L. Heron, P. S. Shaffer, and L. C. McDermott, Student understanding of the ideal gas law, part II: A microscopic perspective, Am. J. Phys. 73, 1064 (2005).

[8] M. E. Loverude, C. H. Kautz, and P. R. L. Heron, Student understanding of the first law of thermodynamics: Relating work to the adiabatic compression of an ideal gas, Am. J. Phys. 70, 137 (2002).

[9] A. D. Robertson, An investigation of university student and $K-12$ teacher reasoning about key ideas in the development of the particulate nature of matter, Ph.D., University of Washington 2011.

[10] S. Carey, Cognitive science and science education, Am. Psychol. 41, 1123 (1986).

[11] M. McCloskey, Naive theories of motion, in Mental Models, edited by D. Gentner and A. Stevens (Erlbaum, Hillsdale, NJ, 1983), p. 299-324.

[12] G. J. Posner, K. A. Strike, P. W. Hewson, and W. A. Gertzog, Accommodation of a scientific conception: Toward a theory of conceptual change, Sci. Educ. 66, 211 (1982).

[13] A. Gupta, A. Elby, and L. D. Conlin, How substance-based ontologies for gravity can be productive: A case study, Phys. Rev. ST Phys. Educ. Res. 10, 010113 (2014).

[14] D. Hammer, A. Elby, R. E. Scherr, and E. F. Redish, Resources, Framing, and Transfer, in Transfer of Learning: Research and Perspectives, edited by J. Mestre (Information Age Publishing, Greenwich, CT, 2005), p. $89-120$.

[15] D. Hammer and A. Elby, Tapping epistemological resources for learning physics, J. Learn. Sci. 12, 53 (2003).

[16] D. Hammer, Student resources for learning introductory physics, Am. J. Phys. 68, S52 (2000).

[17] A. Elby and D. Hammer, On the substance of a sophisticated epistemology, Sci. Educ. 85, 554 (2001).

[18] R. Engle and F. Conant, Guiding principles for fostering productive disciplinary engagement: Explaining an emergent argument in a community of learners classroom, Cognit. Instr. 20, 399 (2002).

[19] B. W. Harrer, Identifying productive resources in secondary school students' discourse about energy, Ph.D., University of Maine, 2013.

[20] A. R. Daane, S. Vokos, and R. E. Scherr, Goals for teacher learning about energy degradation, Phys. Rev. ST Phys. Educ. Res. 10, 020111 (2014).

[21] B. Jordan and A. Henderson, Interaction analysis: Foundations and practice, J. Learn. Sci. 4, 39 (1995).

[22] F. Erickson, Qualitative methods in research on teaching, in Handbook of Research on Teaching, edited by M. C. Wittrock (Macmillan, New York, 1986), pp. 119-161.

[23] L. S. Vygotsky, The development of scientific concepts in childhood: The design of a working hypothesis, in Thought and Language, edited by A. Kozulin (MIT Press, Boston, 1986).

[24] J. Lave, Situating learning in communities of practice, in Perspectives on Socially Shared Cognition, edited by L. B. Resnick, J. M. Levine, and S. D. Teasley (American
Psychological Association, Washington, DC, 1991), p. $63-82$.

[25] A. Sfard, On two metaphors for learning and the dangers of choosing just one, Educ. Res. 27, 4 (1998).

[26] J. V. Wertsch, Mediation, in The Cambridge Companion to Vygotsky, edited by H. Daniels, M. Cole, and J. V. Wertsch (Cambridge University Press, New York 2007).

[27] E. Hutchins, How a cockpit remembers its speeds, Cogn. Sci. 19, 265 (1995).

[28] R. Nemirovsky, C. Rasmussen, G. Sweeney, and M. Wawro, When the classroom floor becomes the complex plane: Addition and multiplication as ways of bodily navigation, J. Learn. Sci. 21, 287 (2012).

[29] R. Stevens, Divisions of labor in school and in the workplace: Comparing computer and paper-supported activities across settings, J. Learn. Sci. 9, 373 (2000).

[30] J.A. Maxwell, Using qualitative methods for causal explanation, Field Methods 16, 243 (2004).

[31] J. A. Maxwell, Causal explanation, qualitative research, and scientific inquiry in education, Educ. Res. 33, 3 (2004).

[32] W. C. Salmon, Causality and Explanation (Oxford University Press, New York, 1998).

[33] National Research Council, A Framework for K-12 Science Education: Practices, Crosscutting Concepts, and Core Ideas (National Academies Press, Washington, DC, 2012)

[34] NGSS Lead States, Next Generation Science Standards: For States, by States (National Academies Press, Washington, DC, 2013).

[35] R. E. Scherr, H. G. Close, E. W. Close, and S. Vokos, Representing energy. II. Energy tracking representations, Phys. Rev. ST Phys. Educ. Res. 8, 020115 (2012).

[36] A. R. Daane, S. Vokos, and R. E. Scherr, Conserving energy in physics and society: Creating an integrated model of energy and the second law of thermodynamics, AIP Conf. Proc. 1513, 114 (2013).

[37] S. B. McKagan, R. E. Scherr, E. W. Close, and H. G. Close, Criteria for creating and categorizing forms of energy, AIP Conf. Proc. 1413, 279 (2012).

[38] R. S. Russ, J. E. Coffey, D. Hammer, and P. Hutchison, Making classroom assessment more accountable to scientific reasoning: A case for attending to mechanistic thinking, Sci. Educ. 93, 875 (2009).

[39] R. S. Russ, R. E. Scherr, D. Hammer, and J. Mikeska, Recognizing mechanistic reasoning in scientific inquiry: A framework for discourse analysis developed from philosophy of science, Sci. Educ. 92, 499 (2008).

[40] P. Machamer, D. Darden, and C. F. Craver, Thinking about mechanisms, Philos. Sci. 67, 1 (2000).

[41] P. Machamer, Activities and causation: The metaphysics and epistemology of mechanisms, Int. Studies Philos. Sci. 18, 27 (2004).

[42] L. Darden and C. F. Craver, Strategies in the interfield discovery of the mechanism of protein synthesis, Stud. Hist. Philos. Biol. Biomed. Sci. 33, 1 (2002).

[43] L. Darden, Strategies for discovering mechanisms: Schema instantiation, modular subassembly, forward/backward chaining, Philos. Sci. (Suppl.) 69, S354 (2002).

[44] N. J. Nersessian, How do scientists think? Capturing the dynamics of conceptual change in science, in Cognitive 
Models of Science, edited by R. N. Giere (University of Minnesota Press, Minneapolis, 1982), p. 3-45.

[45] R. P. Feynman, R. B. Leighton, and M. Sands, The Feynman Lectures on Physics (Addison-Wesley, Reading, MA, 1969).

[46] C. E. Weiman, W. K. Adams, and K. K. Perkins, PhET: Simulations that enhance learning, Science 322, 682 (2008).

[47] L. S. Vygotsky, Internalization of higher psychological processes, in Mind in Society: The Development of Higher Psychological Processes, edited by M. Cole et al. (Harvard University Press, Cambridge, MA, 1978).

[48] A. Gupta, D. Hammer, and E. F. Redish, The case for dynamic models of learners' ontologies in physics, J. Learn. Sci. 19, 285 (2010).

[49] D. Hammer, F. Goldberg, and S. Fargason, Responsive teaching and the beginnings of energy in a third grade classroom, Rev. Sci. Math. ICT Educ. 6, 51 (2012).

[50] L. J. Atkins and B. W. Frank, Examining the products of responsive inquiry, in Responsive Teaching in Science and Mathematics, edited by A. D. Robertson, R. E. Scherr, and D. Hammer (Routledge, New York, 2015).

[51] A. R. Daane, S. B. McKagan, S. Vokos, and R. E. Scherr, Energy conservation in dissipative processes: Teacher expectations and strategies associated with imperceptible thermal energy, Phys. Rev. ST Phys. Educ. Res. (to be published).
[52] D. Hammer and E. van Zee, Seeing the Science in Children's Thinking: Case Studies of Student Inquiry in Physical Science (Heinemann, Portsmouth, NH, 2006), p. 186.

[53] A. D. Robertson, L. J. Atkins, D. M. Levin, and J. Richards, What is responsive teaching?, in Responsive Teaching in Science and Mathematics, edited by A. D. Robertson, R.E. Scherr, and D. Hammer (Routledge, New York, 2015).

[54] B. W. Harrer, V. J. Flood, and M. C. Wittmann, Productive resources in students' ideas about energy: An alternative analysis of Watts' original interview transcripts, Phys. Rev. ST Phys. Educ. Res. 9, 023101 (2013).

[55] D. Hammer, Discovery learning and discovery teaching, Cognit. Instr. 15, 485 (1997).

[56] D. L. Ball, With an eye on the mathematical horizon: Dilemmas of teaching elementary school mathematics, Elem. School J. 93, 373 (1993).

[57] C. Rogers, Significant learning: In therapy and in education, On Becoming a Person: A Therapist's View of Psychotherapy (Houghton Mifflin, New York, 1961), p. 279-296.

[58] S. B. Empson and V. R. Jacobs, Learning to listen to children's mathematics, in Tools and Processes in Mathematics Teacher Education, edited by D. Tirosh and T. Wood (Sense Publishers, Rotterdam, NL, 2008), p. 257.

[59] J. E. Coffey, D. Hammer, D. M. Levin, and T. Grant, The missing disciplinary substance of formative assessment, J. Res. Sci. Teach. 48, 1109 (2011). 